\title{
Development of the Roll Type Incremental Micro Pattern Imprint System for Large Area Pattern Replication
}

\author{
Jung-Han Song ${ }^{1}$, Hye-Jin Lee ${ }^{1}$, Shuhuai Lan ${ }^{1}$, Nak-Kyu Lee ${ }^{1}$, Geun-An Lee ${ }^{1}$, \\ Tae-Jin Lee ${ }^{1}$, Seogou Choi ${ }^{1}$, and Sung-Min Bae ${ }^{2}$ \\ ${ }^{1}$ Manufacturing Convergence R\&D Department, Korea Institute of Industrial Technology, \\ 1271-18 Sa 3-dong, Sangrok-gu, Ansan-si, Gyeonggi-do, 426-173 Korea \\ ${ }^{2}$ Dept. of Industrial \& Management Engineering, Hanbat National University Korea \\ \{Jhsong, naltl, bluetree, nklee, galee, ltj0822, schoi\}@kitech.re.kr, \\ loveiris@hanbat.ac.kr
}

\begin{abstract}
Flexible display has been attracting attention in the research field of next generation display in recent years. And polymer is a candidate material for flexible displays as it takes advantages including transparency, light weight, flexibility and so on. Rolling process is suitable and competitive process for the high throughput of flexible substrate such as polymer. In this paper, we developed a prototype of roll-to-flat (R2F) thermal imprint system for large area micro pattern replication process, which is one the key process in the fabrication of flexible displays. Tests were conducted to evaluate the system feasibility and process parameters effect, such as flat mold temperature, loading pressure and rolling speed. $100 \mathrm{~mm} \times 100 \mathrm{~mm}$ stainless steel flat mold and commercially available polycarbonate sheets were used for tests and results showed that the developed R2F system is suitable for fabrication of various micro devices with micro pattern replication on large area.
\end{abstract}

Keywords: Flexible Display, Roll-to-Flat Thermal Imprint, Micro Pattern Replication, Polycarbonate.

\section{Introduction and Background}

As the semiconductor industry and IC (Integrated Circuit) industry developing and flat panel display market growing there are demands to search a method that can provide production with high precision and good quality at a low-cost and highthroughput way. Among them, Micro- or Nano- structure fabrication or highprecision nanoscale lithography process is the key technology to the manufacturing of photonic components, micro - and nanofluidic chips, chip-based sensors and biological applications. Most manufacturer and researcher are looking for a proper fabrication way. Except for the conventional technologies such as well-known Optical lithography and Electronic Beam lithography, several alternative approaches towards nanostructure fabrication have been exploited, for example, Micro-contact Printing, Nanoimprint Lithography, Scanning-Probe-Based techniques, Dip-Pen Lithography and Nanoplotting, as well as Stenciling. [1-3]. Among them Nanoimprint lithography 
(NIL) was considered widely as the potential high-throughput, high-resolution and low-cost manufacturing method. [4]

Early in 1995, S.Y. Chou et al. firstly proposed the first Nanoimprint Lithography process in NanoStructure Laboratory of University of Minnesota. They firstly realized patterning sub-25 $\mathrm{nm}$ and sub-10 nm nanostructures in the polymers on a silicon substrate, which is followed by Reactive Ion Etching (RIE) or dry etching process. During the process, a pattern mold was pressed onto a thin thermoplastic polymer film on a substrate to create vias and trenches with a minimum size of $25 \mathrm{~nm}$ and a depth of $100 \mathrm{~nm}$ in the polymer. [4-5]

The research on the NIL has spread over the world and distinguished results have been achieved. By now, they had generated many kinds of NIL technologies. Generally speaking we can classify them into three categories of technology: (1) Hot-Embossing Nanoimprint Lithography (HE-NIL) [4,5] (2) UV-type Nanoimprint Lithography (UV-NIL) [6-8] (3) Soft-Lithography [9]. Now the three kinds of NIL technologies have developed more and have been used widely in many different applications, such as biomedical product [10], data storage [11], optical parts [12], organic electronics [13] and so on. However, there are still many problems should be solved in the conventional NIL process so that it can be used as mass production method in the industry. One of the most important problem is that it cannot significantly improve the throughput in the patterning of large area product with low cost because it is not a continuous process.

To overcome this problem Roller-type Nanoimprint Lithography (RNIL) [14] was firstly proposed by S.F. Chou et al. and has developed quickly and become the potential manufacturing method for industrialization of nanoimprinting process, due to its prominent advantage of continuous process, simple system construction, highthroughput, low-cost and low energy consuming. For the conventional flat nanoimprint, the whole area of the sample is imprinted at the same time. But for RNIL, only the adjacence area of contact line is pressed for a given time, significantly reducing the effects of thickness unevenness and dust from environment. So in this article, our aim is to give a brief review on the state-of-the-art of the novel NIL process.

\section{Continuous Roller-Type Nanoimprint Lithography Process}

Actually Roller-type manufacturing (Roll to Roll) methods has previously used in many industrial fields, such as gravure printing or flexography printing (or flexo). It was used to imprint the pattern of the roller on flexible thin films. They were traditionally used for printing newspapers, magazines and packages. Especially in the field of manufacturing for flexible electronics, this fabrication method has already developed. Flexible electrophoretic displays manufactured by roll-to-roll (R2R) processes have been demonstrated good reliability and performance. [15] Wang Xiaojia et al. have also developed a novel full color electrophoretic film manufacturing process using Roller-type method. [16] In the actual industrial manufacturing, typical R2R manufacturing consists of three essential steps, which are deposition, patterning and packaging, as shown in Fig. 1. [17-18]. 
As its name implying, Roller-type manufacturing method has the advantage of providing a continuous and high throughput. When it was novelly used in the nanoimprint lithography technology, the cost of NIL production can be reduced.

Early in 1998, Hua Tan et al. firstly proposed and demonstrated an alternative approach to the NIL process, the Roller-type Nanoimprint Lithography process. In this process, they had achieved some sub-100 nm resolution patterns transfer. [14] The schematic of this method is shown as Fig. 2. Here the roller-type nanoimprint lithography process system mainly consists of three function components: a roller, a movable platform, and a hinge. They developed two methods for RNIL: (a) rolling a cylindrical mold on a flat and solid substrate; (b) putting a flat mold directly on a substrate and rolling a smooth roller on the back of the mold as show in fig. 4 . In the first method, the cylindrical mold has thin metal film mold around a smooth roller. On the thin metal sheet, micro patterns were formed For example the master mold made of $100 \mu \mathrm{m}$ thick of Ni with 700-nm - wide tracks was used for a compact disk. In both methods, the roller temperature was set above the glass transition temperature $\mathrm{Tg}$ of the resist, PMMA (Polymethyl Methacrylate) in Fig. 3, while the temperature of the platform is set below Tg. Therefore, only the area in contact with the roller has a temperature higher than $\mathrm{Tg}$, This makes the resist in the area molten and imprinted with the patterns. This is different from flat nanoimprint, where the entire resist, heated above $\mathrm{Tg}$, was imprinted simultaneously and the pressure is applied until the resist is cooled down and cured.

Evolving these two methods, many kinds of Roller-type imprint lithography process have been developed We categorize the Roller-type NIL as methods, using roller mold and using flat mold. In the following, some typical and distinguished research will be introduced.

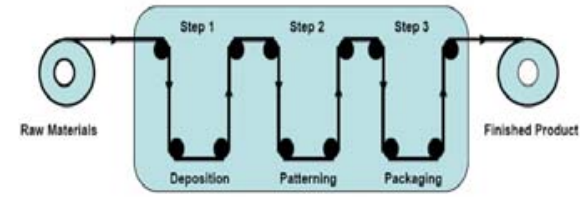

Fig. 1. Three steps in industrial R2R process

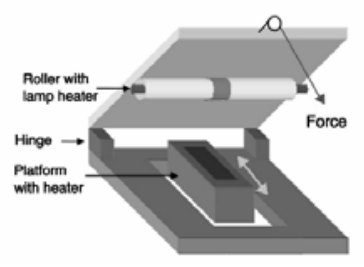

Fig. 2. Schematic of a roller nanoimprint system

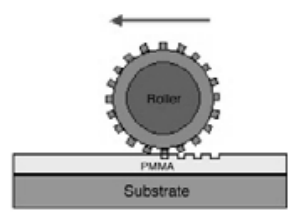

(a)

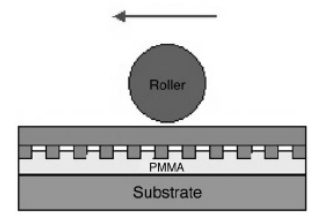

(b)

Fig. 3. Two methods of RNIL: a) using roller mold; b) using flat mold \& smooth roller 


\subsection{RNIL Process Using Roller Mold}

A roller pattern mold is widely used in RNIL. It can be used both in thermal type (Hot-Embossing) and UV type (UV-based) RNIL process. In the thermal type RNIL process, by rotating and pressing the cylinder mold into the polymer film coated on the substrate with the temperature over glass transition $(\mathrm{Tg})$, the feature on the mold is transferred to the film and the film is pushed backward at the same time. By cooling down, the transferred pattern is cured. While in the UV type RNIL process, the UV light is illuminated when the rotating roller mold contacts with the coated film. The original phase of film is gel or liquid. By illuminating the light, the phase chenged to solid and then the feature on the roller mold is replicated as shown in fig. 4.

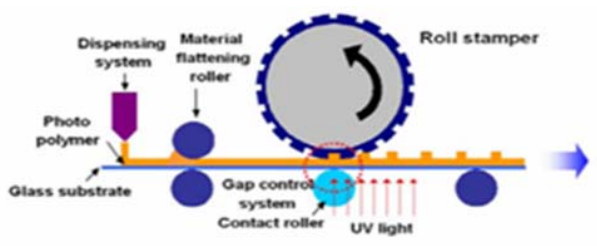

Fig. 4. Schematic diagrams of the UV-type RNIL system for rigid substrate

No matter in Thermal type or UV type RNIL process, the fabrication of roller mold is important, as it directly involves the replication quality of the imprinting process. The manufacturing accuracy of the mold decides the resolution of the RNIL product. And it also acts as a barrier of the development of the RNIL technology. As the target feature size gets smaller below several tens of micrometers, it is difficult to fabricate. To create the pattern feature on the surface of the mold cylinder, researcher have searched several methods. Generally speaking, there are mainly two methods to fabricate roller pattern mold. One is directly fabricating the micro/nano feature on the cylinder surface, using ultra-precision machining or other manufacturing method. [19] The other is to cover a shim stamper around the cylinder surface, in which the shim stamper is fabricated previously using electron beam lithography or other lithography technologies. [20]

\subsection{RNIL Process Using Flat Mold and Smooth Roll (R2F)}

Comparing with RNIL using roller mold, the RNIL using flat mold (R2F Process) is only being studied by few researchers. The reason mainly lies in that it cannot realize the continuous replication. The gap controlling between smooth roll and flat mold is additionally required. To pattern the feature repeatedly as a continuous process, the transportation of the roller or the flat mold should also be embodied precisely as well as the rotation of the roller. But using flat mold in RNIL has its advantage on mold fabrication, including fabrication accuracy and cost, as it has been at a mature level of micro/nano feature manufacturing. The feature can be formed using the fabrication methods such as MEMS, ultra-precision machining, electron beam maching or photolithography. 


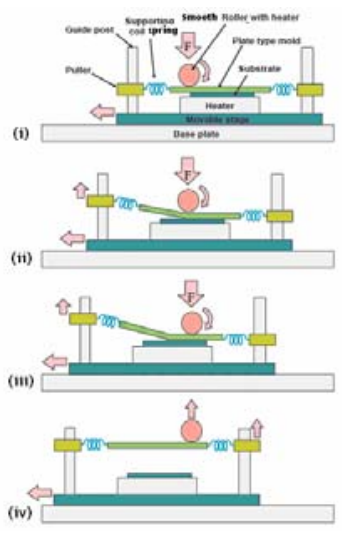

(a)

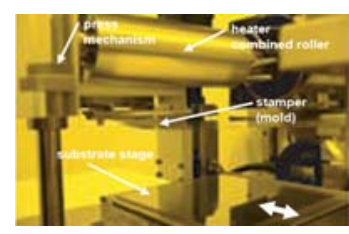

(b)

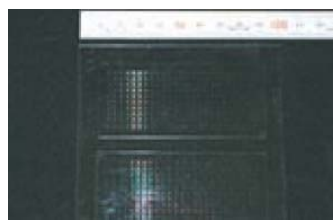

(c)

Fig. 5. (a) RNIL system concept using flat mold and smooth roller from AIST; (b) Picture of the real equipment; (c) Imprinting result on a flexible substrate

Several research group proposed concepts using smooth roller and flat mold in their process. AIST(Advanced Industrial Science and Technology) in Japan proposed a process for large area replication. The configuration is shown as Fig. 5. Micro/nano feature on the mold can be replicated to a polymer substrate by applying force and heat with a heater combined in the roller. An automatic mold releasing mechanism is installed in the system to avoid transferred pattern deformation error by mismatch of thermal expansion ratio between a mold (metal) and a substrate (polymer). The distinguished advantage is combining the smooth roller and a movable stage that contains the substrate and a flexible plate mold. By pressing the smooth roller to the flexible plate mold, the pattern can be transferred to the substrate, at the same time the rotation of the roller push the movable stage backward. During the process, smooth roller and substrate were heated by their heater elements respectively. Good result was claimed in their report, as shown in the Fig. 5. (c).

\section{R2F Imprint System Development}

In this study, we developed a prototype of lab-scale thermal RNIL equipment using flat mold (R2F System) based on the survey. A photograph of the R2F system is shown as Fig. 6. It is made up of several major sub-units, including roller imprint unit, polymer holding and auto-releasing unit, control unit and PC unit for process parameters input (temperature, speed, force, etc.) and result data acquisition.

Fig. 7 shows the photographs of the R2F micro thermal imprint system consisting of major components such as the pressing roller with heaters inserted into the core, which is actuated by AC-servo motor, the movable stage, flat mold combined with a heater, and polymer substrate holding and auto-releasing device. The polymer substrate supply and holding device is made up of a pair of linear grippers and a pair 


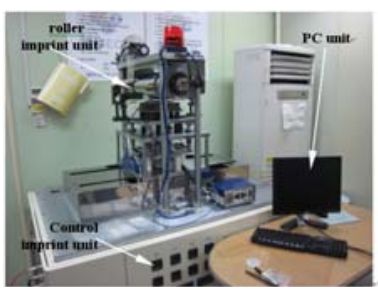

Fig. 6. Overview of the developed system

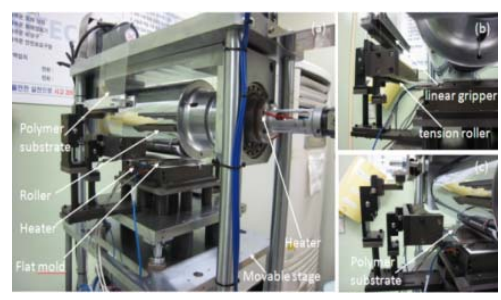

Fig. 7. Photographs of major components

Table 1. Specifications of the roll-to-flat micro thermal imprint system

\begin{tabular}{l|l|c}
\hline \multicolumn{2}{l|}{ System size $(\mathrm{mm})$} & $1500 \times 1100 \times 1800$ \\
\hline \multicolumn{4}{c}{ Stage stroke $(\mathrm{mm})$} & 700 \\
\hline \multirow{4}{*}{ Roller } & Diameter $(\mathrm{mm})$ & 160 \\
\cline { 2 - 3 } & Width $(\mathrm{mm})$ & 290 \\
\cline { 2 - 3 } & Maximum temperature $\left({ }^{\circ} \mathrm{C}\right)$ & 200 \\
\cline { 2 - 3 } & Maximum loading pressure (tonf) & 1 \\
\cline { 2 - 3 } & Scanning speed $(\mathrm{mm} / \mathrm{s})$ & $0.1-10$ \\
\cline { 2 - 3 } & Material & Stainless steel \\
\hline \multirow{4}{*}{ Flat Mold } & Maximum mold area $\left(\mathrm{mm}^{2}\right)$ & $30 \times 150$ \\
\cline { 2 - 3 } & Maximum temperature $\left({ }^{\circ} \mathrm{C}\right)$ & $0.1-10$ \\
\cline { 2 - 3 } & Moving speed $(\mathrm{mm} / \mathrm{s})$ & \\
\hline
\end{tabular}

of tension rollers. This device can adjust the small gap between polymer substrate and flat mold. When the pattern replication is being performed in the semi-linear contact area, other area of polymer will not contact with the mold. After rolling and the mold moving forward, the replication area completed will automatically be separated from the mold. The concept is introduced because the imprinted pattern can be released vertically without damage from the flat mold since the continuous rolling movement.

The movable stage, supporting the flat mold, can move parallel to the sample surface in one direction, at the speed range from $0.1 \mathrm{~mm} / \mathrm{s}$ to $10 \mathrm{~mm} / \mathrm{s}$. The maximum distance allowed for the movable stage is about $500 \mathrm{~mm}$. The maximum press force for the pressing rolling device is $500 \mathrm{kgf}$ with a precision of less than $1 \mathrm{~N}$. The detailed specification of the system is summarized in Table 1 .

A stainless steel flat mold with size of $100 \mathrm{~mm} \times 100 \mathrm{~mm}$ was used in the experiments, which was fabricated by dicing process. The entire surface of the mold is composed with square micro structure arrays with height of $90 \mu \mathrm{m}$, width of 200 $\mu \mathrm{m}$ and $110 \mu \mathrm{m}$ spacing. Fig. 8 shows a photograph of the flat mold that is embedded on the movable stage. Fig. 9 illustrates a typical 3D scanning image of a mold sub-feature using whitelight scanning interferometry (SNU Precision, Korea) Commercial polycarbonate(PC) substrates were imprinted in the tests. Under the process condition that flat mold temperature is above PC's glass transition temperature $\left(150{ }^{\circ} \mathrm{C}\right)$ and low rolling speed, the micro patterns on the flat mold were transferred to the PC substrates. 


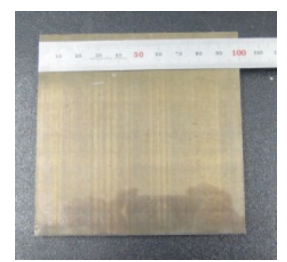

Fig. 8. Photograph of the flat mold prepared

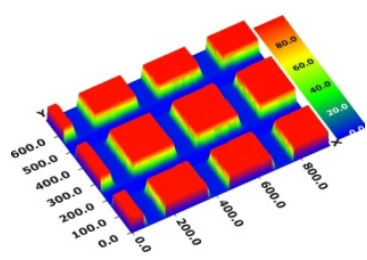

Fig. 9. Scanning 3D image of mold sub-feature

\section{Result and Discussion}

In order to evaluate the performance and feasibility of the developed R2F micro thermal imprint system and to study the rolling process parameters, such as imprinting temperature, loading pressure and rolling speed, several experiments were conducted.

Using the whitelight scanning interferometry the tomograph of the micro pattern on the flat mold and the imprinted pattern on the substrate surface were measured and compared. Based on these measured results, the evaluation of process formability was carried out.

Trial tests were conducted using a rigid and thick PC substrate with thickness of 2 $\mathrm{mm}$. Fig. 10 and 11 shows an example of replication result from flat mold to PC substrate. The process was conducted under the condition of $200 \mathrm{kgf}$ of press force, 1 $\mathrm{mm} / \mathrm{s}$ rolling speed (also the moving speed of movable stage) and $160{ }^{\circ} \mathrm{C}$ temperature of flat mold. The roller was not heated and it was in room temperature. From the result shows that good replication results of uniform micro cup arrays were fabricated over the whole surface area. Some defects on the beginning part (on the bottom of the figure) due to the change of rolling speed from 0 to the constant speed $2 \mathrm{~mm} / \mathrm{s}$ at the beginning of process.

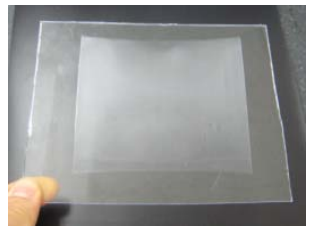

Fig. 10. Photograph of the imprinted substrate

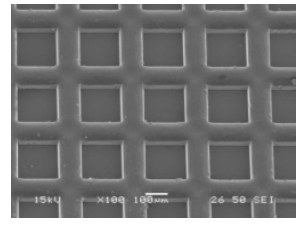

Fig. 11. SEM image of imprinted patterns

\section{Conclusion}

In summary we have developed a prototype of lab-scale roll-to-flat (R2F) thermal imprint system for large area micro pattern replication process as a potential application of flexible display based on the survey. Series of tests were carried out using a stainless steel flat mold at size of $100 \mathrm{~mm} \times 100 \mathrm{~mm}$ and commercial available polycarbonate (PC) substrates to evaluate the system feasibility and process control parameters effect, such as flat mold temperature, loading pressure and rolling speed. The test results show that the developed R2F system is suitable for fabrication of micro devices with micro pattern replication at large area. 


\section{Acknowledgement}

This work has been financially supported by Korea Research Council for Industrial Science \& Technology through the project of Convergence Manufacturing Technology Development of Bio-Medical Applicable Systems for Geriatric Diseases (B551179-09-02-00). The authors are grateful to the colleagues for their essential contribution to this work.

\section{References}

1. Jay Guo, L.: Nanoimprint Lithography: Methods and Material Requirements. Advanced Material 19, 495-513 (2007)

2. Gates, B.D., et al.: New Approaches to Nanofabrication: Molding, Printing, and Other Techniques. Chemical Reviews 105, 1171 (2005)

3. Sotomayor Torres, C.M., et al.: Nanoimprint lithography: an alternative nanofabrication approach. Materials Science and Engineering C 23, 23-31 (2003)

4. Chou, S.Y., et al.: Imprint of sub-25 nm vias and trenches in polymers. Applied Physics Letters 67(21), 3114-3116 (1995)

5. Chou, S.Y., Krauss, P.R.: Imprint Lithography with Sub-10 nm Feature Size and High Throughput. Microeleetronic Engineering 35, 237-240 (1997)

6. Bender, M., et al.: Fabrication of nanostructures using a UV-based imprint technique. Microelectronic Engineering 53, 233-236 (2000)

7. Otto, M., et al.: Characterization and application of a UV-based imprint technique. Microelectronic Engineering 57-58, 361-366 (2000)

8. Colburn, M., et al.: Step-and-flash Imprint Lithography: A New Approach to High Resolution Patterning. In: Proc. of SPIE, vol. 3676, p. 379 (1999)

9. Xia, Y., Whitesides, G.M.: Soft Lithography. Angewandte Chemie International Edition, vol. 37, pp. 550-575 (1998)

10. Pepin, A., et al.: Nanoimprint lithography for the fabrication of DNA electrophoresis chips. Microelectronic Engineering 61-62, 927-932 (2002)

11. Nilsson, M., Heidari, B.: Breaking the Limit-Patterned Media for 100 Gbits and beyond. Obducat, Malmo, Sweden

12. Park, Y.K., Kostal, H.: Nano-Oprics Redenfine Rules for Oprical Processing. Communication System Design, 23-26 (August 2002)

13. Clavijo Cedeno, C., et al.: Nanoimprint lithography for organic electronics. Microelectronic Engineering 61-62, 25-31 (2002)

14. Tan, H., Gilbertson, A., Chou, S.Y.: Roller nanoimprint lithography. Journal of Vacuum Science \& Technology B 16(6), 3926-3928 (1998)

15. Hou, J., et al.: Reliability and performance of flexible electrophoretic displays by roll-toroll manufacturing processes. In: SID 2004 Digest, Seattle, USA, pp. 1066-1069 (2004)

16. Xiaojia, W., HongMei, Z., Li, P.: Roll-to-roll manufacturing process for full color electrophoretic film. In: SID 2006 Digest, San Francisco, CA, pp. 1587-1889 (2006)

17. Grawford., G.P., et al.: Roll-to-Roll Manufacturing of Flexible Displays. Flexible Flat Panel Displays, 410-445 (2005)

18. Schwartz, E.: Roll to Roll Processing for Flexible Electronics. Cornell University MSE 542: Flexible Electronics, May 11 (2006)

19. Ahn, S., et al.: Continuous ultraviolet roll nanoimprinting process for replicating largescale nano- and micropatterns. Applied Physics Letters 89, 213101 (2006)

20. Makela, T., et al.: Continuous roll to roll nanoimprinting of inherently conducting polyaniline. Microelectronic Engineering 84, 877-879 (2007) 